\title{
Aportaciones al análisis constructivo de fábricas antiguas: La capilla de La Antigua de San Miguel en Morón (1538)
}

\author{
Contributions to the building analysis of old constructions: \\ The chapel of La Antigua at San Miguel, Morón (1538)
}

F. Pinto $^{(*)}$

RESUMEN

Las obras de restauración sobre construcciones antiguas es un momento indispensable para el conocimiento del legado constructivo que permanece oculto en ellas, sobre todo cuando son resultado de reformas y ampliaciones históricas. El conocimiento de las soluciones en muros, arcos y bóvedas de piedra adoptadas por los constructores, la lógica constructiva seguida y las técnicas aplicadas, es indispensable para comprender su comportamiento estructural, las causas de las lesiones perceptibles, y proceder a su correcta evaluación. Durante la restauración de la capilla de La Antigua, adosada al templo de San Miguel en Morón, hemos podido observar este legado y confirmar hasta que punto era necesario su conocimiento interrelacionando diversas fuentes como la información obtenida mediante su levantamiento gráfico, la lectura de la secuencia constructiva, los estudios geotécnicos y documentales, así como el análisis de los sistemas gráficos de control formal que permitieron su trazado, conocidos por tratados y manuscritos de Arquitectura.

$148-170$

Palabras clave: Restauración; construcción; cantería; bóvedas.

\section{SUMMARY}

Carrying out restoration works at old constructions offers a valuable opportunity for learning more about the building legacy they conceal, especially when they are the result of historical remodellings and extensions. A knowledge of the solutions that the builders adopted for stone walls, arches and vaults, of the construction logics followed and of the techniques applied provides vital clues for understanding their structural behaviour and the reasons for the discernible lesions, and for evaluating their condition correctly. During our restoration of the chapel of La Antigua adjoining the church of San Miguel in Morón, we were able to observe this legacy and confirm the importance of compiling knowledge obtained from various sources such as the graphic survey of the construction, the building sequence, geotechnical and documentary studies, and the analysis of the formal graphic control systems used to draw the plans, known as architecture treatises and manuscripts.

Keywords: Restoration; construction; stonework; vaults. 


\section{ESTADO DE LA CUESTIÓN}

El desarrollo de una restauración donde se acometen trabajos de registro y refuerzos estructurales con desmontajes parciales o totales de sus elementos constructivos, es un momento especial para el conocimiento de las numerosas soluciones tecnológicas y materiales que han quedado ocultas en sus fábricas.

Este legado oculto es una fuente de información tan importante como vulnerable, pues en muchas ocasiones son amortizadas por operaciones realizadas desde una perspectiva contemporánea imponiendo su propia lógica constructiva. El conocimiento y puesta en valor de estos elementos ocultos tiene ya una larga tradición. Desde los trabajos de August Choisy el papel histórico de la construcción adquirió carta de naturaleza, formando parte así de los valores propios de la Arquitectura (1). Desde entonces se ha favorecido la comprensión de nuestro patrimonio arquitectónico como resultado de un proceso homogéneo donde ha destacado la unidad formal y constructiva frente a la complejidad de su evolución en el tiempo. Sin consideramos esto último, es decir, las posibles reformas y transformaciones sufridas por los edificios históricos, el análisis de los sistemas constructivos y sus procesos se hace más complejo y ambiguo. En este sentido son destacables las aproximaciones a la lectura de esta complejidad desde el ámbito de la arqueología, recurriendo a métodos científicos que intentan descifrar la secuencia temporal en la que se han producido. Considerar este enfoque puede afectar de forma sustancial a la comprensión del comportamiento de sus materiales, la respuesta estructural de sus fábricas o el funcionamiento de sus sistemas de instalaciones. Según los casos, puede llegar a ser tan decisivo conocer los valores universales del edificio (artísticos, históricos, tipológicos, testimoniales o rememorativos), como las condiciones específicas en las que se han desenvuelto a lo largo del tiempo (alteraciones del terreno, restos sobre los que se asientan, configuración específica de las reformas, materiales y recursos locales empleados, contexto profesional, social y funcional, etc).

Más allá de documentar o describir un caso específico, esta aportación pretende enfocar y subrayar la necesidad de una lectura detenida de las huellas que ha dejado el tiempo en cada uno de estos edificios, entenderlos como artefactos que surge de una cultura, unos conocimientos y unas formas de hacer a veces muy alejados de los actuales, e interpretarlos conforme a las claves que nos aporta cada época y lugar. Recordando el párrafo con el que Choisy comienza su Historia de la Arquitectura (1), "Los monumentos de la arquitectura naciente nos advierten en su manifestación más simple esos inevitables lazos que unen el modo de construir a los estados sucesivos de la Humanidad y hacen de la Historia del Arte un resumen de la Historia misma de las sociedades." A esto añadimos, siguiendo a otros investigadores (2), aqueIla vieja idea de Vitruvio de la dificultad de separar en el proyecto arquitectónico los aspectos puramente técnicos o funcionales de los específicamente formales.

Este es el caso de la capilla de Nuestra Señora de la Antigua en la Iglesia de San Miguel Arcángel de Morón de la Frontera, una pequeña edificación levantada para alojar una advocación familiar, que se construye adosada a uno de sus muros laterales. Un ejercicio compositivo en el que se muestra claramente los problemas asociados a las reformas de fábricas en piedra en un momento especialmente significativo de nuestra historia, situado en el tránsito entre las arraigadas tradiciones constructivas del tardogótico y las innovaciones formales y tecnológicas del renacimiento. Este momento tan especial ha sido estudiado en profundidad desde diversos enfoques: En cuanto a los cambios en la estereotomía de la piedra son determinantes los trabajos de Palacios (3), Gómez (4) y Rabasa (5) que han dado lugar a un proyecto de investigación financiado por el Ministerio de Ciencia e Innovación (6). Dentro de este mismo enfoque es de destacar la reciente aportación de Palacios y Bravo (7) sobre la experiencia de construir un modelo reducido de una bóveda artesonada muy parecida a la que presentamos en esta aportación. Sin embargo son muy escasos los testimonios donde se muestran piezas originales o la estructura interna de las bóvedas que analizamos, de ahí la importancia de dar a conocer sus peculiaridades. Otro enfoque se plantea al considerar la relación de estos cambios con los conocimientos científicos y tecnológicos coetáneos a este tipo de soluciones constructivas, en este caso centrándonos en el ámbito territorial del arzobispado hispalense. La revisión y recuperación de la visión perspectiva y la proyección cónica que fue llevada a cabo por los cosmógrafos de la Casa de la Contratación de Sevilla en las primeras décadas del siglo XVI, para mejorar la elaboración de la cartografía, influirán de manera determinante en la forma de trazar bóvedas y de representar edificios, y especialmente en los sistemas de control formal de las bóvedas (8). Finalmente, si nos referimos a los datos historiográficos sobre los maestros implicados en este momento de transfor- 
mación, a los que haremos referencia en los apartados siguientes, debemos destacar el estudio monográfico recientemente realizado por Rodríguez Estévez sobre el maestro Martín de Gainza (9) donde se muestran las numerosas obras que trazó y ejecutó a lo largo del arzobispado hispalense, territorio al que dedicó su etapa profesional más importante. También debemos citar por su importancia en el tema tratado, el trabajo que actualmente está desarrollando el grupo de investigadores liderado por la profesora Alonso, dedicado al estudio de las relaciones transfronterizas entre maestros del tardogótico hispano (10).

\section{LA IGLESIA DE SAN MIGUEL Y LA CAPILLA DE LA ANTIGUA}

La iglesia de San Miguel de Morón de la Frontera es el resultado de un dilatado proceso constructivo donde se muestra con nitidez la búsqueda de la unidad del conjunto frente a la diversidad de las etapas constructivas que la historiografía nos ha dejado documentadas (11). Es un edificio de tres naves y amplio crucero, todos abovedados, resultado de la profunda reforma de un templo previo gótico-mudéjar, que sufrió un importante derrumbe de su techumbre a finales del s.XV. La reconstrucción comenzó con el nuevo siglo y lo hizo por los pies, sucediéndose una serie de fases constructivas que fueron cerrando sus naves hasta interrumpirse la obra a la altura del crucero en 1539. El resultado de estas primeras obras es un conjunto de cuatro tramos de naves cubiertos por bóvedas de nervios diagonales, terceletes y combados de diversa configuración, que descargan sobre arbotantes y estribos rematados por pináculos exteriormente, así como en pilares baquetonados entre las naves laterales y central, y ménsulas en los encuentros con los paramentos interiores. El crucero y la cabecera resultan de una reforma posterior de un ábside poligonal cuyos restos probablemente podamos registrar en la intervención que emprenderemos en el próximo mes de la presentación de esta aportación. El actual está compuesto por dobles tramos simétricos de bóvedas baídas con capillas adosadas en los brazos y cúpula en la nave central, percibiéndose con claridad la evolución formal y constructiva introducida a partir de la segunda mitad del s. XVI (12). El conjunto conserva un complejo conjunto de restos: algunos muros mudéjares originales realizados con fábrica de ladrillo y mampuestos irregulares, fábricas pétreas del tardogótico y renacimiento, para finalizar con obras anexas como sacristías y sagrarios, portadas y retablos barrocos, donde la tecnología constructiva es más compleja y heterogénea (Figura 1).
Podríamos definirla como una iglesia parroquial de estructura basilical, a la que se añadió un amplio crucero, peculiaridad tipológica en este ámbito territorial que demuestra su rango prioral o de iglesia mayor. Los edificios parroquiales solían trazarse libres de capillas anexas, salvo aquellas heredadas de alguna fundación que permanecía en uso y que estaban obligados a respetar. Habitualmente los nuevos anexos surgían cuando algún promotor de la nobleza o aristocracia local mostraba interés en adquirir uno de los espacios aledaños al templo para levantar una capilla funeraria, o conmemorar alguna advocación vinculada a su historia familiar. Por lo general, esta operación requería de importantes modificaciones de la estructura mural del templo, que debía horadarse para comunicar estas nuevas fundaciones con el espacio de las naves y hacerse así visible. Las condiciones técnicas para unir y abrir estas construcciones al templo eran consultadas a su maestro mayor, quien daba las pautas necesarias para garantizar la estabilidad y seguridad del edificio principal, inspeccionaba el terreno y establecía los límites a las obras.

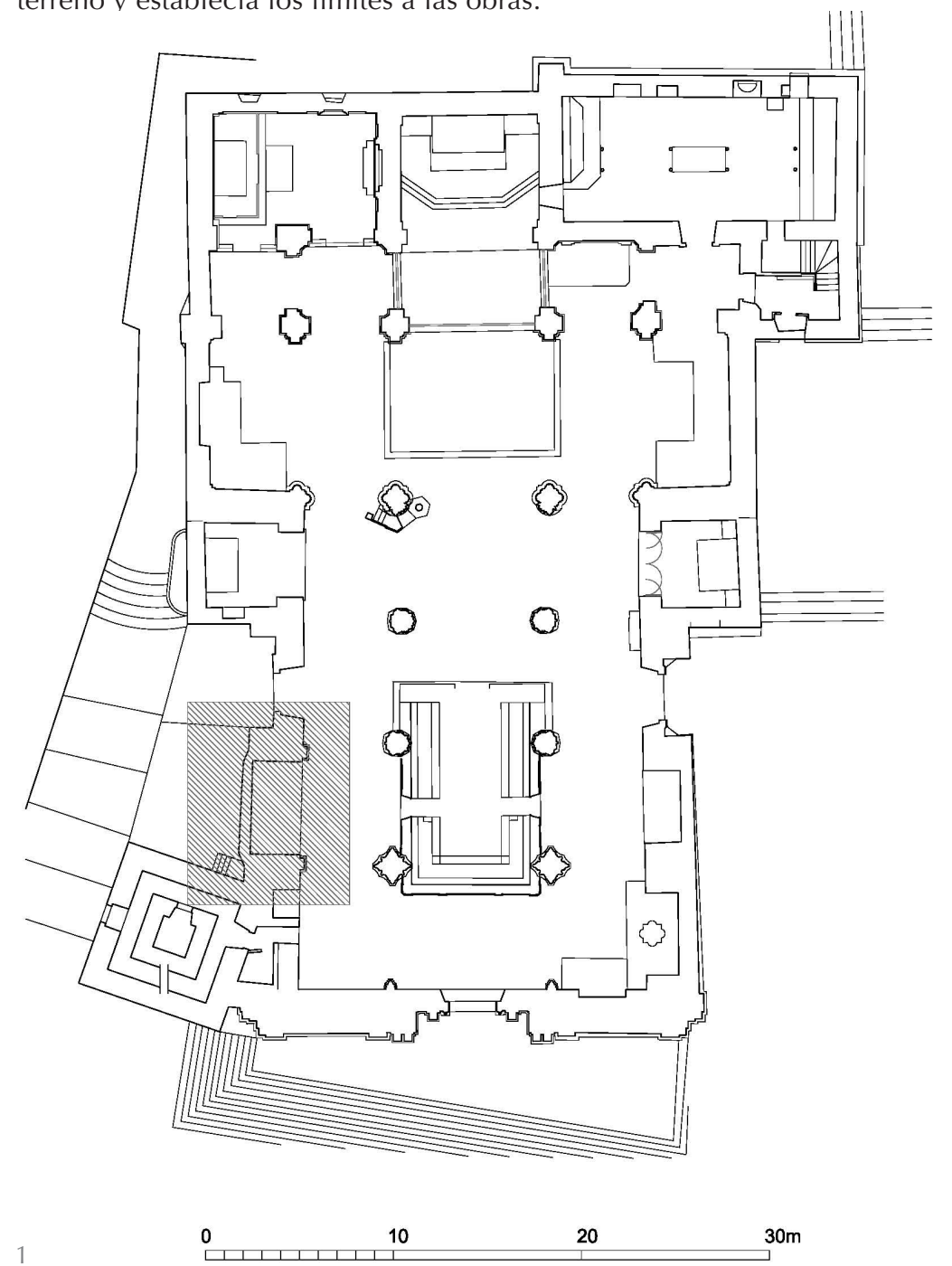

1. Planta de la iglesia de San Miguel.

(Dibujo A. Almagro 1998) 
2. Vista del cuerpo de la nave hacia los pies.

(Foto autor 2010)

3. Vista de la capilla de la Antigua desde el interior del templo.

(Foto autor 2012

4. Vista exterior de la capilla de la Antigua.

(Foto autor 2012)
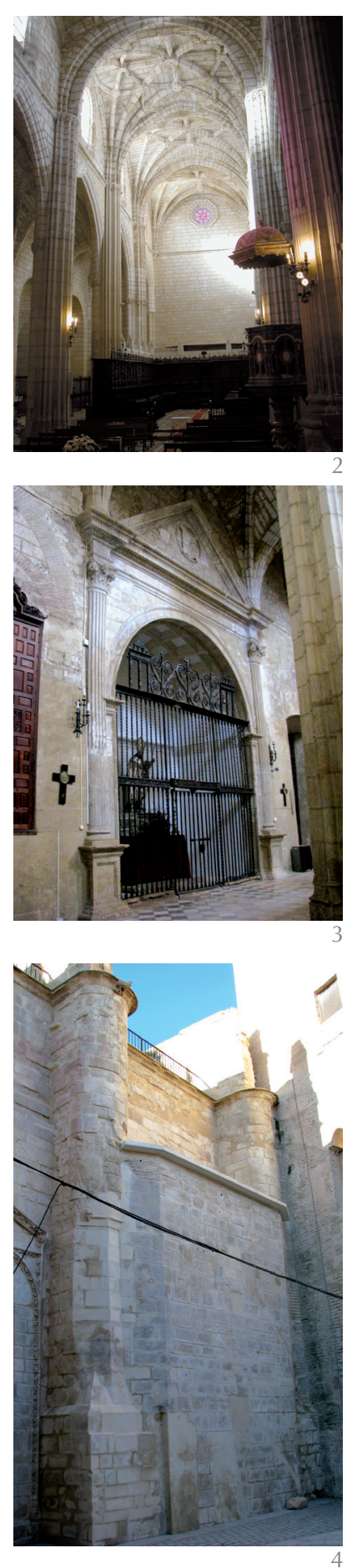

Este modo de proceder suele conocerse a través de oficios y requerimientos notariales de cesión de derechos y exposición de condiciones, o por contratos e informes de los maestros, donde se muestra el cuidado puesto en aclarar cuestiones que eran a la vez técnicas, funcionales y estéticas. Las primeras destinadas a preservar la estructura del edificio principal, y las restantes a garantizar la armonía formal con el conjunto. Las soluciones constructivas resultantes son consecuencias del encuentro entre la búsqueda de la configuración deseada para la capilla, los límites constructivos del templo que las recibe, las condiciones de los lugares disponibles y los materiales y los conocimientos técnicos disponibles. Lugar común que ha demostrado, en muchos de los casos documentados, la puesta en práctica de importantes dosis de ingenio expresado en asombrosas y vistosas soluciones formales y en inimaginables decisiones constructivas, muchas de las cuales suelen quedar ocultas. Por citar sólo algunos casos, todas aquellas construcciones de sacristías levantadas en edificios mayores, colegiatas y catedrales durante el s. XVI, o las numerosas ampliaciones que se suceden casi de forma cotidiana en los monasterios, organismos vivos que se adaptan al devenir de las ordenes religiosas.

En el caso de la iglesia de San Miguel, esto sucede en cada uno de los tramos de sus naves (Figura 2). Merece la pena detenerse brevemente en el relato de estas etapas, pues ofrecerá algunas referencias importantes para entender el momento de construcción de la capilla. La historia de esta reforma comienza en 1506 por los pies del templo gótico-mudéjar original, siguiendo las pautas formales y constructivas de la catedral hispalense (13). La dirección de las obras corría entonces a cargo de Anton Ruiz, un maestro vinculado a la finalización de la magna hispalense, buen conocedor de la escuela constructiva allí nacida a mediados del siglo anterior. Los primeros pilares y bóvedas siguen los modelos desarrollados en la catedral, adaptados a las dimensiones y materiales aquí disponibles. Los dos siguientes tramos fueron realizados entre 1528 y 1534 bajo las órdenes de Diego de Riaño, maestro mayor de la catedral y el arzobispado y desde 1523 maestro bajo el mecenazgo de los condes de Ureña, uno de los mecenas más importantes del templo moronense. Este arquitecto fue uno de los introductores de las innovaciones formales y constructivas propias del primer renacimiento en el ámbito hispalense, como demostró en el proyecto del Cabildo Civil y la Sacristía Mayor de la Catedral de su capital. A pesar de ello, se vio obligado a continuar éste y otros edificios conforme al lenguaje gótico con el que comenzó la reforma, a los que fueron introduciendo las novedades características del tardogótico castellano. Este maestro compatibilizaba su presencia en numerosas obras repartidas por Sevilla, su extenso arzobispado y en ciudades tan lejanas como Peñafiel o Valladolid en las que trabajó por intermediación de su mecenas. Esta dispersión de obras pudo ser abordada, no sin problemas, gracias a que dispuso de un extenso taller de oficiales y maestros como el documentado en San Miguel. A este taller perteneció Martín de Gainza como aparejador entre 1529 y 1534, quien a la muerte del maestro, fue el encargado de cerrar muchas de las obras comenzadas entre las que estaba la ahora descrita. Posteriormente, en torno a 1550 vuelva a documentarse su presencia, en este caso como nuevo maestro mayor. De nuevo, la razón de esta tardanza en asumir las obras de San Miguel se debió a los encargos en la capital. Una vez incorporado a éste cargo se documenta cerrando la última bóveda del cuerpo de la nave principal y abriendo los cimientos para el nuevo crucero que ampliaría notablemente el templo.

En el margen de tiempo que permaneció el edificio inactivo (1534-1550) se fundó la capilla tratada por deseo de doña Mencia de Osorio Morejón, dedicándola a la Virgen de la Antigua, advocación marinera que se relaciona con la profesión de su esposo Juan Pérez de Osma, navegante que participó con Pizarro en la conquista de Perú. Se construyó en 1538 bajo trazas de Martín de Gainza, antiguo aparejador de la fábrica, ya por entonces maestro mayor de la catedral hispalense.

La capilla es un pequeño espacio de cinco metros de amplitud por dos metros de profundidad que se levantó apurando el espacio entre los dos estribos del tercer tramo de la nave principal, con el objetivo de quedar abierto en todo su ancho a la nave lateral, a modo de capilla hornacina (Figura 3 y 4). La portada que lo enmarca fue articulada por Gainza mediante un gran arco de medio punto entre semicolumnas corintias resaltadas por pilastras de fondo que quedan rehundidas en los sillares de piedra. El orden arquitectónico queda rematado por un entablamento clásico y un gran frontón, configurando uno de los ejemplos en lenguaje "a lo romano" más logrados en ese momento inicial de su producción como maestro. El modelo lo encontramos en otras obras de Gainza como la portada de la torre fachada de la iglesia de Santa María de Utrera, o a una escala reducida casi de maqueta, usada como benditera en las estancias laterales de la Capilla Real (Figura 5). A diferencia de estos casos, el espacio de la capilla 
no está cubierto por un capialzado o una venera, sino por una bóveda baída artesonada rectangular. Se completa el espacio con tres paramentos lisos recorridos por una pequeña cornisa que sirve de imposta para la bóveda y una reja que protege la propiedad. Esta obra la podemos relacionar con otras fábricas trazadas por Riaño y aparejadas por Gainza en la catedral hispalense, claras exponentes del primer renacimiento, coetáneas e incluso algo anterior a los modelos gráficos que nos ofrece Diego de Sagredo en el folio $3 \mathrm{v}$ de la edición de la Medidas del Romano (1549). Posteriormente, los elementos usados en esta capilla se verán reflejados en otros realizados por Gainza en su papel de maestro mayor, como es el caso la citada Capilla Real levantadas entre 1535 y 1557, año de su fallecimiento. Es, por tanto, un maestro que tuvo que trabajar conforme al tardogótico cerrando fábricas antiguas, y con los nuevos requerimientos formales del renacimiento en fundaciones reales, capitulares y de la aristocracia local.

\section{CONSTRUCCIÓN DE LA CAPILLA}

Considerar el espacio de la capilla supone no sólo indagar sobre su configuración formal o su cronología, sino plantear el hecho mismo de ser una reforma o ampliación, y por tanto, valorar la modificación de un estado previo tanto de equilibrio estructural como del sistema constructivo que lo materializa. Esto es, el hecho de abrir este espacio al templo mediante el vaciado del muro entre los dos estribos aprovechando los laterales de estos últimos como paramento de la misma, implicó una operación de gran envergadura. En realidad, los únicos elementos constructivos aportados por el proyecto de Gainza son un muro de fondo, una bóveda, y el trasdosado parcial de los paramentos de los estribos. Un esfuerzo por minimizar materiales y recursos tecnológicos que fue llevado hasta el extremo, ocasionando una cierta debilidad de todo el tramo de la iglesia donde se ubica (14), lo que nos vuelve a recordar el difícil equilibrio entre los requerimientos técnicos y artísticos que citamos al inicio de esta aportación. Este precario equilibrio se verá sólo compensado por la posterior construcción de una pequeña edificación exterior para cuarto de las deshoras o del cura semanero, que usará el muro de cierre de la capilla y los estribos como elementos sustentante de sus alfarjes de madera. Las numerosas reformas realizadas en esta edificación anexa, incluida su sustitución por una vivienda de VPO en los años setenta del pasado siglo, y su posterior demolición en esos mismos años siguiendo una de esas operaciones de sventramento que tanto daño han causado a nuestro patrimonio, produjeron impor- tantes deformaciones que afectaron definitivamente al ya alterado tramo de la nave, requiriendo el apuntalamiento exterior del muro de fondo de la capilla y un apeo de la bóveda mediante una densa estructura de madera que impedía el acceso a su interior. Esto ocurría en 1998, salvándolos de la ruina (Figuras 6 y 7 ).

La evolución de este conjunto de edificaciones pudimos documentarla a través de una campaña de trabajos previos consistentes en: un análisis arqueológico de sus paramentos y subsuelo exterior de la capilla, varios estudios geotécnicos y de materiales, así como un primer estudio de su estabilidad estructural a partir de los indicios exteriormente visibles y los datos obtenidos de los ensayos, apoyados por un levantamiento fotogramétrico (15). A pesar de estos trabajos, quedaron importantes lagunas por cubrir, pues no pudimos acceder a la cubierta de la capilla ni a su interior, al estar cuajado de puntales de madera improvisados con cierta urgencia y acierto ante el peligro de derrumbe. Esta circunstancia hizo que el análisis de esta estructura no se pudiera completar hasta que se iniciaron los trabajos de intervención.

\section{EL CONOCIMIENTO A TRAVÉS DE LA INTERVENCIÓN}

Las incertidumbres planteadas obligaron a la planificación de una serie de registros y tomas de datos durante los trabajos

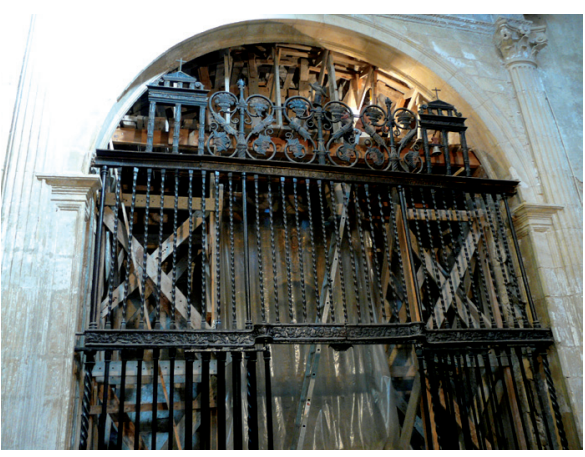

6

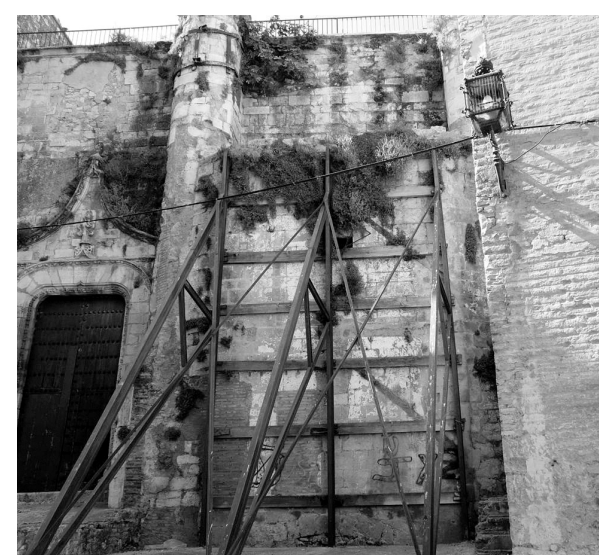

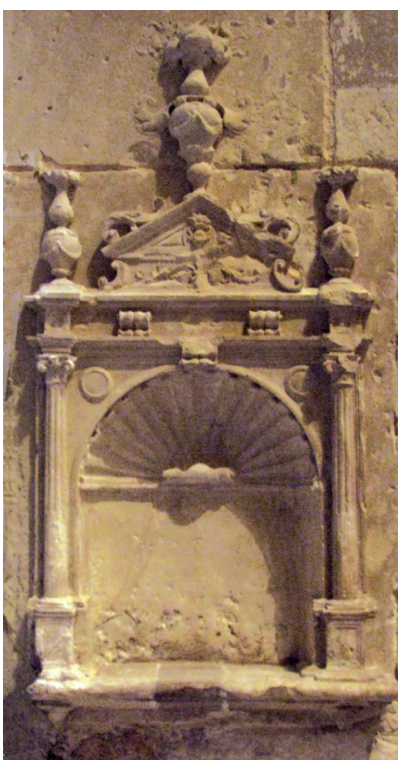

5

5. Benditera de la Capilla Real, Catedral de Sevilla.

(Foto autor 2012)

6. Vista de la capilla apuntalada desde el interior del templo.

(Foto autor 2008)

7. Vista de la capilla apuntalada desde el exterior.

(Foto autor 2008) 
8. Cata en el interior del templo junto a la capilla.

(Foto autor 2010

9. Arco de descarga de la cimentación.

(Foto autor 2009)

10. Sección transversal y longitudinal de la capilla.

(Dibujo autor 2009)

11. Planta de la capilla con fases constructivas.

(Dibujo autor 2010).

12. Perspectiva de la capilla. Elementos constructivos. (Foto autor 2012) de intervención que ha dado origen a las reflexiones que ahora se exponen. Este trabajo consistió en una captura métrica y fotográfica de cada nuevo elemento oculto, su inventario y posterior elaboración de un modelo gráfico que nos permitió comprender el problema en conjunto, y adaptar las acciones inicialmente proyectadas.

La excavación de dos catas exteriores e interiores a la capilla permitió contar con varios datos de interés. En primer lugar que el terreno estaba formado por un extenso estrato de rocas de yeso a 1,46 metros bajo la cota del suelo interior del templo, verificando así la información obtenida mediante dos testigos sacados previamente (16) (Figura 8). Este estrato es el firme donde se construye parte de la cimentación del templo formada por mampuesto de piedra calcarenita y restos cerámicos. La pendiente del terreno que descendía hacia el norte por la calle aledaña, quedaba nivelada por este cimiento formando así el subsuelo del templo. En segundo lugar, la zona donde se asienta la capilla, que queda fuera de esta nivelación, estaba ocupada por enterramientos que correspondían a la fundación del primer templo gótico mudéjar, lo que implicaba la existencia de un suelo removido y renovado, presentando una escasa cohesión y resistencia. En tercer lugar, comprobamos que la cimentación de la capilla prácticamente no existía. El único elemento nuevo que necesitaba cimentarse era el muro de fondo, y para ello se aprovechó la cimentación de los estribos del templo mediante la creación de un arco de descarga muy rebajado, ejecutado directamente sobre el relleno de tierra exterior (Figura 9). Está formado por sillares irregulares que se ajustaron de forma muy desigual a la geometría que requiere el dovelaje, pues parecen proceder de materiales y piezas reaprovechadas de la antigua fábrica. La construcción de este arco se ejecutó de forma separada y paralela a la antigua cimentación del muro del templo que aún se conserva en el subsuelo de la capilla como único testigo de la configuración inicial de la fachada tardo-

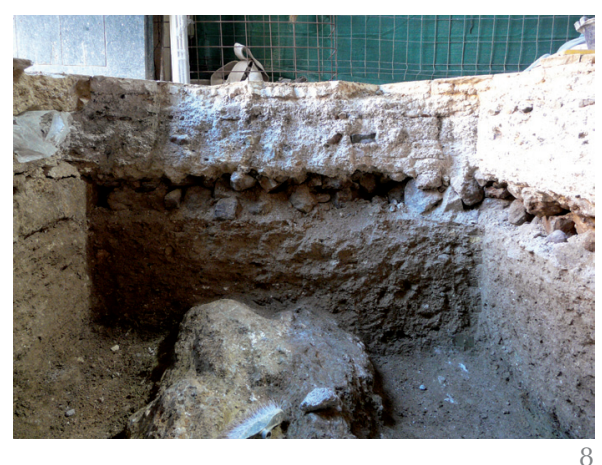

gótica. La imposición del arco se produce en puntos muy bajos de la cimentación de los estribos, garantizando así la estabilidad necesaria mediante su empotramiento con el terreno, siempre y cuando no se alterase su estratigrafía, circunstancia que se produjo muchos años después.

A pesar de esta solución y del escaso espesor del muro de la capilla, unos 0,45 metros, su paramento exterior quedó levemente adelantado respecto a los estribos. Este desfase se explica, sin duda, por la necesidad de conseguir una proporción conforme para la bóveda que la cubre. Con tal fin se manipuló la profundidad, ya que su ancho quedó condicionado por el vano entre los estribos. Nuevamente una razón compositiva condiciona la solución constructiva, hasta el punto de generar una traba muy débil entre este muro de fondo y la fábrica de los estribos.

Los paramentos laterales se obtuvieron mediante la modificación de los estribos. Estos últimos presentan una configuración muy peculiar, donde se alternan geometrías cuadradas, achaflanadas y cilíndricas, una composición que en la actualidad ha quedado sumamente alterada por la erosión y las reformas sufridas. La base del estribo presenta un pronunciado resalte a modo de podio, que provoca dos planos distintos en sus laterales. Para conseguir la unidad vertical en las paredes laterales de la capilla se aplacaron los estribos a partir de la altura del podium, a una altura de 2,60 metros respecto al suelo de la iglesia. La variación entre el aparejo original y el aplacado se perciben en el desajuste entre las hiladas de los sillares. Al nivelarse en vertical se facilitaba también la recepción de los arcos formeros de la bóveda sobre el aplacado, evitando así penetrar en el estribo. En estos arcos murales están labradas las piezas de encuentro con la red de nervios de la bóveda, lo que le confiere un apoyo continuo en toda su longitud. Esta ausencia de traba la pudimos observar a través de las grandes grietas producidas por el desgarrarse del aplacado a causa del desplome sufrido (Figura 10).

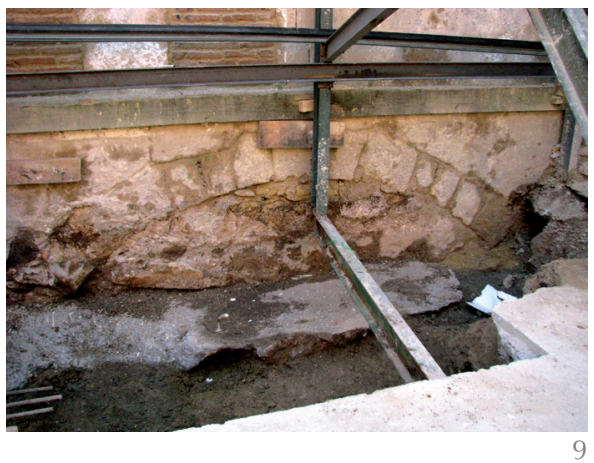



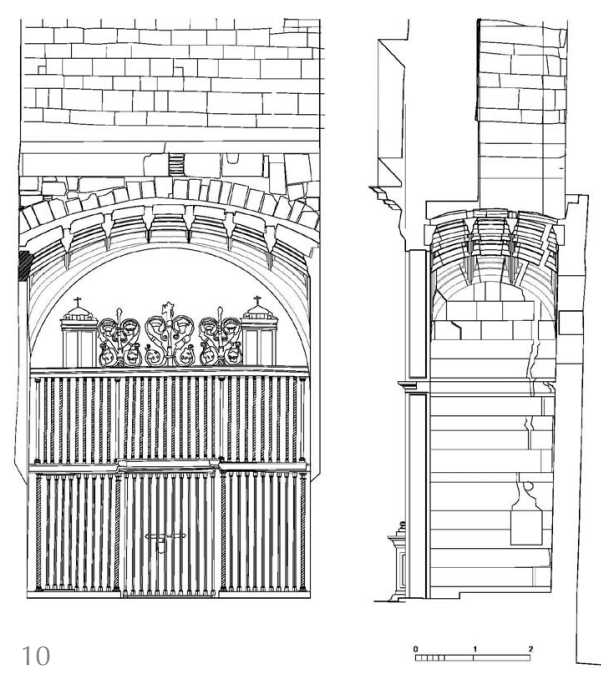

10

Por otro lado, este aplacado debía solventar también el cierre y la traba de la sección del muro resultado del vaciado del arco de la portada. Este registro nos permitió explicar el modo en que estaba ejecutada la unión entre ambas fábricas, y el estado en el que se encontraba. El muro que se vacío para la capilla formaba parte del cierre de las naves laterales del templo y está constituido por dos hojas de fábrica pétrea, la exterior de sillares bien aparejados y la interior de mampuestos, entre las que se realizó un vertido de restos cerámicos y mampuestos de calcarenita amalgamados con cal y áridos. Al producirse el vaciado del muro tan cercano al punto de unión con los estribos, la trabazón quedó seriamente afectada, confiándola ahora al nuevo aplacado. Dado que pudimos medir directamente el espesor de estas piezas y su penetración en el muro, comprobamos que su despiece y trazado estaba más condicionado por las molduras y los elementos formales que formaban la portada, que por aportar un trabado con la antigua fábrica (Figura 11). La profundidad de estos sillares era semejante a la de los restantes materiales usados en el edificio hasta ese momento. Al labrar la decoración de los órdenes arquitectónicos que presentaba un juego de profundidades en sus jambas, quedó muy reducida su sección resistente.

Para valorar la solución descrita hasta este momento proponemos varias consideraciones. En primer lugar, los recursos disponibles cuando se construyó la capilla eran escasos. Entre 1535 y 1543 se suceden varios pleitos entre la población de Morón y los condes de Ureña sobre los derechos adquiridos por tierras y lugares de la población, con no pocos conflictos que debieron repercutir en la paralización de la fábrica del templo desde la muerte de Diego de Riaño. Esta situación debió limitar la extracción de piedra, recurriendo a materiales normalizados y de uso más común con formatos más homogéneos. En segundo lugar, debemos considerar la
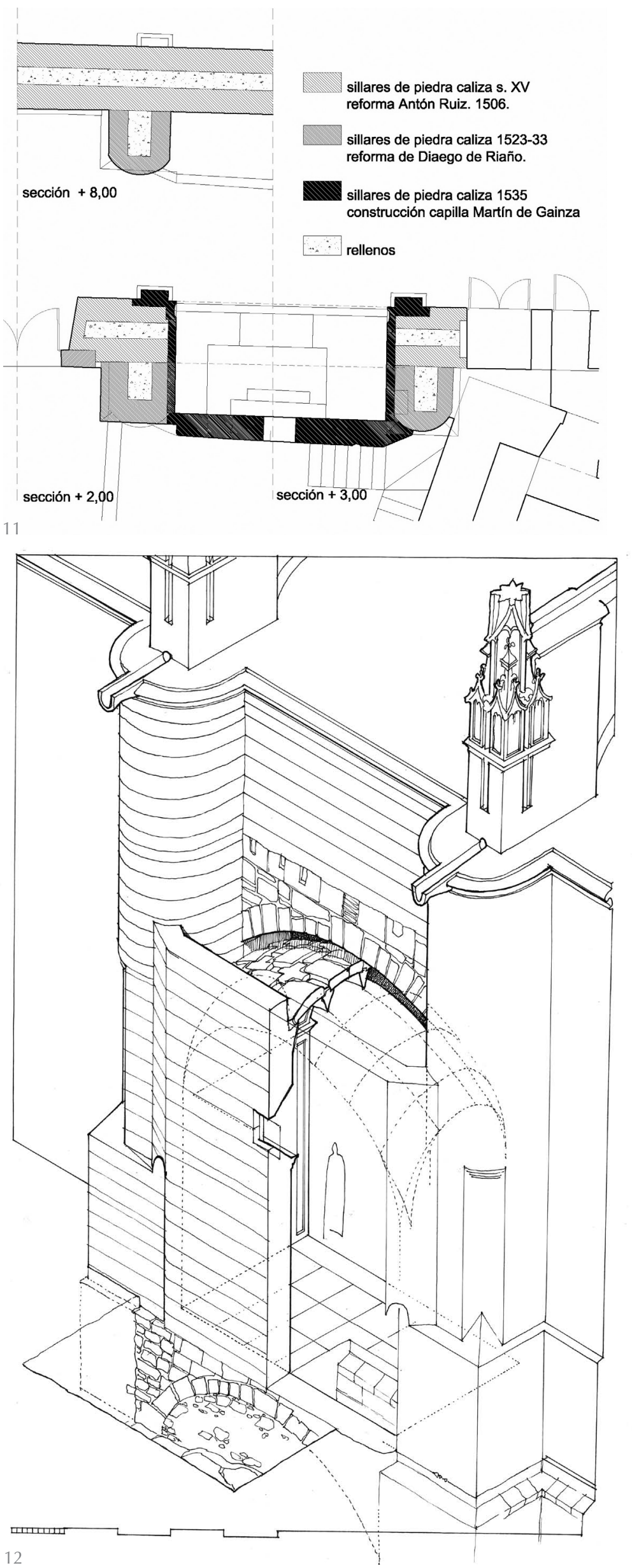
13. Dolios del relleno del trasdós de la bóveda. (Foto autor 2010).

14. Vista del trasdós de la bóveda una vez limpio. (Foto autor 2010).

15. Arco de descarga sobre la bóveda. (Dibujo autor 2010).

16. Enjarje de la bóveda con el arco de embocadura de la capiIla. (Foto autor 2010).
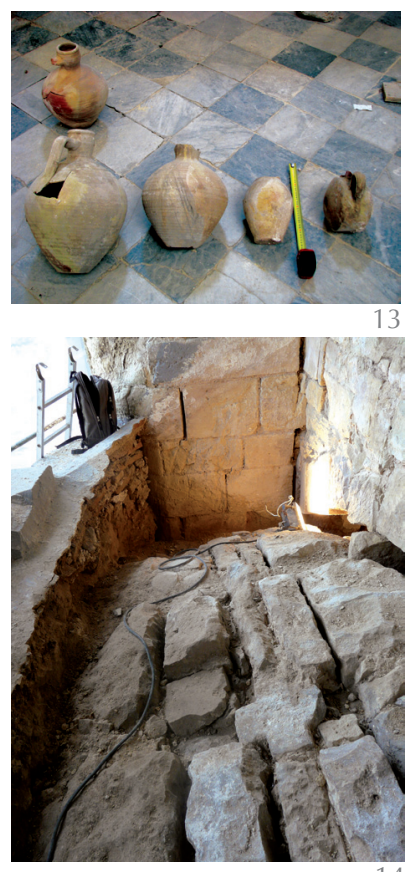

14

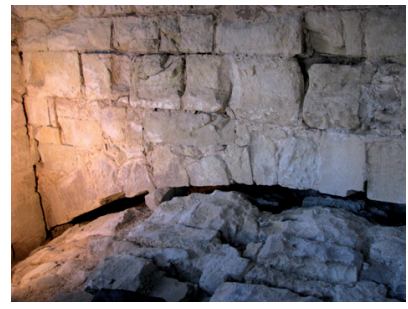

15

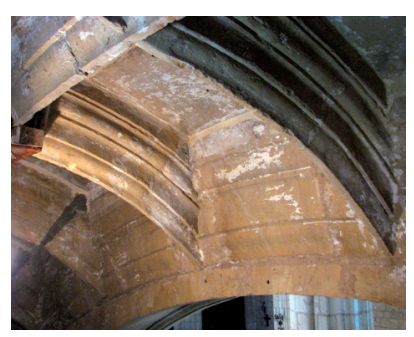

16 dificultad en la ejecución del vaciado de un muro de esta envergadura $(1,25$ metros de espesor) en un punto tan comprometido como la unión con los estribos. En tercer lugar, la labra y montaje de la portada es un proceso complejo, dado el ajuste necesario para responder a un sistema de proporciones "a lo romano", mucho más rígido que el medieval. En este caso, la portada, aunque muestra una cierta libertad compositiva característica de un primer momento de adecuación al nuevo lenguaje, requiere de un gran cuidado en el uso de la medida, lo que probablemente hizo perder algo de atención a la solución constructiva de los complejos encuentros que ocultaban (Figura 12).

Solventadas las jambas y el orden de las pilastras, fue necesario realizar el gran hueco rematado por un medio punto, solucionar la descarga de la parte superior del muro sin afectar a la bóveda de aristas del tramo de la nave lateral vecina. En principio, por los indicios externos, parecía que el propio arco ornamental de la capilla asumía ese papel, pero su potencia es menor que el espesor del muro, haciendo que el resto de la sección cargara, aparentemente, sobre la propia bóveda de la capilla. Esta solución nos planteaba una cierta contradicción, pues la parte de la bóveda que sobresale por el exterior, tendría un nivel de carga muy distinto, siendo a priori muy desfavorable para su correcto funcionamiento estructural. Pero este era nuestro único indicio hasta que no realizamos el registro de su trasdós con objeto de solventar el desajuste que presentaban sus piezas de piedra por efecto de los desplazamientos que había sufrido el conjunto.

La cubierta estaba formada por un relleno de dolios o loza quebrada cuya tipología nos sitúa en un marco temporal coherente al que estamos manejando (17) (Figura 13). Este tipo de piezas cerámicas tiene como misión última, facilitar la formación de una cubierta plana o levemente inclinada sobre la superficie convexa de la bóveda sin gravar excesivamente las cargas sobre ella. Las cubiertas aterrazadas son características de la arquitectura gótica y tardogótica en el ámbito mediterráneo, donde el clima permite evitar el uso de estructuras complementarias de madera y tejas (18). El relleno se produce depositando las piezas de alfarería ordenadas por tamaños con el fin de ocupar el máximo volumen posible sobre la bóveda, con las bocas hacia abajo para evitar la acumulación de agua por condensación en su interior. Cuando a pesar de este apilamiento los espacios entre las piezas eran grandes, se cubría con tejidos ásperos o mallas de cañas sobre las que se tendían capas de mortero de cal y arena que nivelaban la superficie para recibir una solería de ladrillos cerámicos que conseguían una aceptable estanqueidad. Cuando el espacio final entre los dolios era pequeño, se vertía directamente este mortero espeso hasta conseguir la superficie deseada. En la catedral hispalense encontramos magníficos ejemplos de estos rellenos que adquieren variadas soluciones, que son buenos indicadores cronológicos de las diferentes etapas de la obra (19). Encontrar esta solución nos indica que no habían sido removidas con anterioridad, y también que recurrieron a una técnica anclada aún en la tradición constructiva medieval. Nuevamente una arraigada tradición aplicada a nuevas solicitaciones formales.

Retirados estos dolios se detectó la existencia sobre la bóveda de un nuevo arco de descarga en el muro. Este arco quedaba separado del trasdós de la bóveda, y sólo una pequeña deformación introdujo pequeños apoyos que fueron solventados por la nervadura de la bóveda (Figuras 14 y 15). Su apariencia es semejante a la del arco de cimentación aunque de mayor envergadura. Igual que aquel, estaba realizado para quedar oculto, pues sus piezas eran reaprovechadas, ajustándose al despiece radiado de los lechos de cada dovela. Esto supone que la bóveda funciona sin vínculo con el muro, de forma autónoma y homogénea en toda su superficie, algo que solucionaba una de nuestras dudas previas. Por otro lado, se verifica que su ejecución fue posterior a la apertura del muro, es decir, que fue montada una vez el arco de descarga estuvo acabado y el muro vaciado. Sin embargo, la bóveda debió realizarse a la vez que el arco de la embocadura de la capilla hacia la iglesia, pues los arranques de los nervios de la primera están labrados en las dovelas de las segundas (Figura 16). Trabajar libre de las cargas del muro supone una gran ventaja para la ejecución de piezas que requieren de un preciso ajuste, además de ofrecer un funcionamiento más coherente a pesar de estar ubicado un tercio de la superficie de la bóveda bajo el muro. Esta intromisión de la bóveda en el espesor del muro nos remite nuevamente a la cuestión de la proporción. La potencia del arco de embocadura de la capilla está en función de la dimensión frontal y de las pilastras decoradas que forman las jambas del hueco. Al ajustar proporcionalmente esta dimensión, ganaron profundidad para la capilla, lo que permitió poder ajustarla al avance entre los estribos manteniendo la proporción adecuada para el artesonado de la bóveda. Nos movemos nuevamente en un difícil equilibrio entre aspectos compositivos, sistemas de proporciones y necesidades constructivas. 


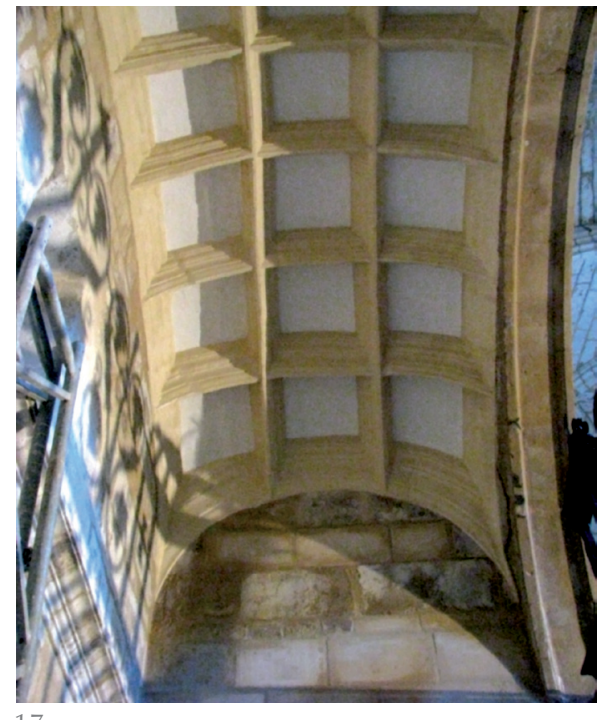

17

Por último abordamos el análisis de la bóveda, en la que encontramos un claro ejemplo de las soluciones artesonadas por cruceros que adquieren gran difusión en el arzobispado tras el paso del maestro Diego de Riaño (Figura 17). Podemos ver ejemplos muy parecidos en las capillas del presbiterio de la Sacristía Mayor y Patio de los Óleos de la Catedral y en la sala capitular del Cabildo Civil y en las primeras obras del monumental claustro del monasterio de San Jerónimo de la capital hispalense, en la capilla de la Vela de la iglesia mayor de Lebrija, o en las sacristías de la iglesia mayor de Aroche y de la prioral de Santa María en Carmona. Al ser una de sus primeras obras, Gainza recurre a una solución aprendida de Riaño. Al buscar otras obras posteriores del mismo autor, observamos como fue introduciendo ciertas modificaciones compositivas que las hacen evolucionar y diferenciarse del tipo de despiece propuesto por Riaño (8), perdiendo esa apariencia aristada de los nervios góticos, a favor de una mayor presencia del nervio. En estas primeras experiencias, a pesar de adquirir una configuración formal totalmente nueva, la respuesta constructiva usó nuevamente la tradición constructiva gótica. Merece la pena, por tanto, detenerse en el análisis de este tipo de elementos y relacionarlo con los elementos detectados hasta el momento.

Aparentemente la bóveda es una superficie baída, alusión a los modelos formales italianos, pero en este caso no existe una superficie activa única, sino una red de líneas que hacen visibles el sistema de proporciones dispuesto para el espacio de la capilla (Figura 18). En este caso la proporción es $7 / 3$, visible gracias a los casetones dispuestos sobre la superficie, son cuadrados en su proyección sobre el plano horizontal. Las líneas de esta cuadrícula están formadas por piezas de piedra cuya sección es semejante a los nervios de una bóveda tardogótica, esto es, en forma de lanceta acabada en un minúsculo plano que forma la arista más exterior. Observándolas de cerca se aprecia como estos nervios tienen sus "moldes revirados" es decir, asimétricos respecto a su eje vertical en función de su posición en la bóveda. En el tratado de trazas y cortes de montea de Alonso de Vandelvira, en los folios $98 \mathrm{v}$. y $99 \mathrm{r}$ se recogen este tipo de despiece de cantería bajo el nombre de capilla perlongada por cruceros (20) (Figura 19). Siguiendo las pautas marcadas en las páginas de este manuscrito, el trazado de los nervios se realizaba sobre el plano horizontal, que en nuestro caso está situado sobre la pequeña cornisa que recorre los tres paramentos de la capilla y se une a la composición de la portada. Posteriormente se levantan "a plomo", esto es, perpendicularmente al plano horizontal, los puntos de cruce entre las líneas de la cuadrícula hasta la altura correspondiente al arco que forma cada línea (21) (Figuras 20 y 21). Sin lugar a dudas, la pieza más compleja es el crucero, elemento que recoge dos curvas distintas pertenecientes a planos verticales perpendiculares entre sí. Esta peculiar y compleja forma estereotómica pretende hacer perceptible la geometría de la bóveda desde el suelo, esfuerzo que ocasiona una importante complejidad en su labra pues

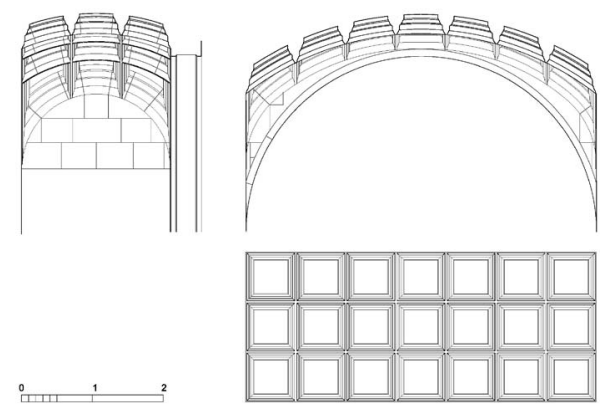

18

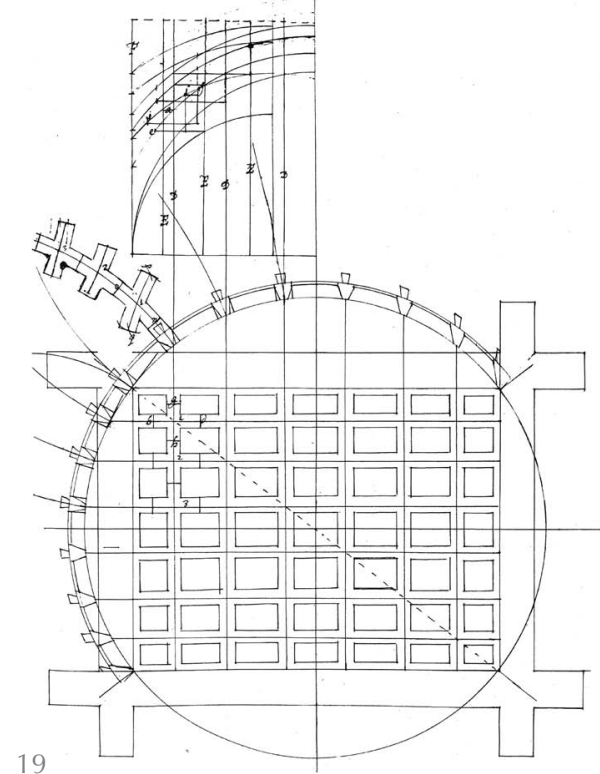

17. Vista interior de la bóveda una vez restaurada.

(Foto autor 2010)

18. Proyecciones ortogonales de la bóveda.

(Dibujo del autor 2010)

19. Folio 98v y 99r del Manuscrito de Alonso de Vandelvira: Capilla perlongada por cruceros. (llustración de la edición consultada) 
20. Imagen de un crucero de la bóveda.

(Foto autor 2010)

21. Proyecciones ortogonales de una pieza del crucero.

(Dibujo autor 2012)

22. Vista del molde de un nervio de la bóveda.

(Foto autor 2010)

23. Vista del nervio con curvatura y resalte superior.

(Foto autor 2010)
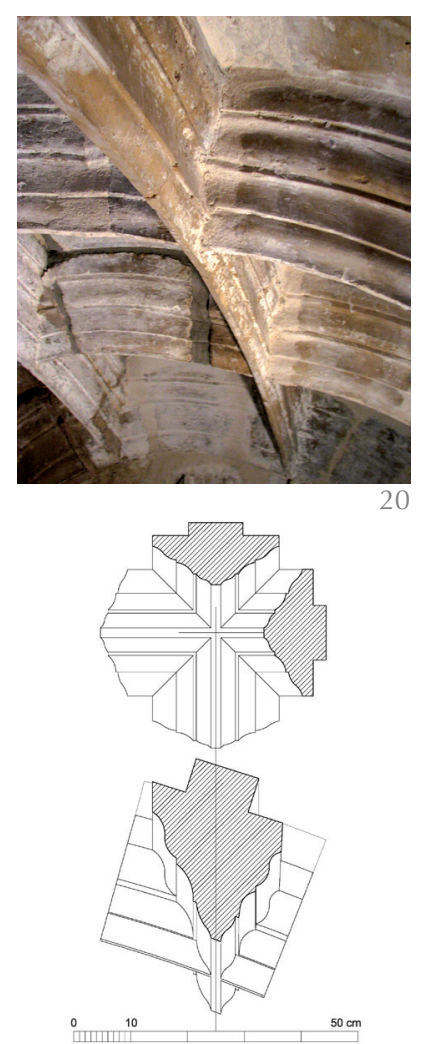

21
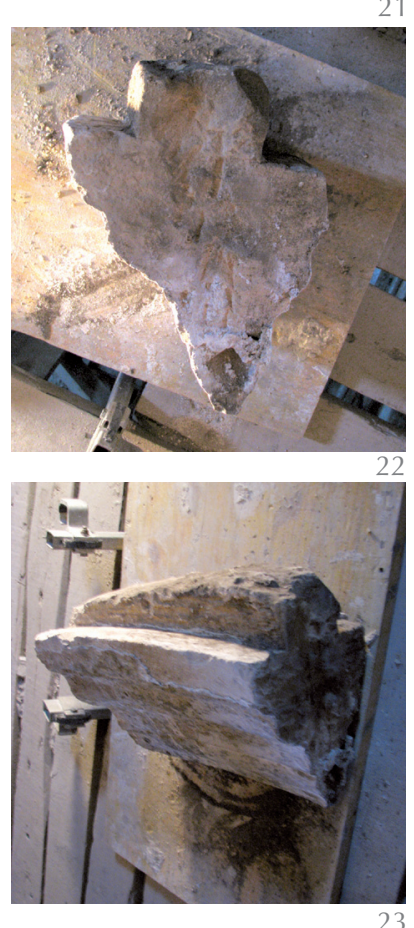

cada elemento es distinto de los restantes. Nuevamente una razón estética origina la puesta en funcionamiento de recursos técnicos muy especializados con resultados formales aparentemente sencillos.

Al trabajar durante la intervención en el intradós, para retacar las piezas sueltas antes de proceder al desmontaje de la cubierta exterior y limpiar su trasdós, pudimos extraer una de las dovelas lineales que estaba suelta, comprobando hasta que punto se asemeja a las descritas en el tratado de Vandelvira (Figuras 22 y 23). Pudimos medirla con exactitud y comprobar la existencia del cordón superior que permite la traba con el tablero que cierra cada casetón, verificando de nuevo el valor del manuscrito consultado como minuciosa recopilación de una tradición constructiva muy experimentada, conocida y usada en el contexto del arzobispado hispalense durante la primera mitad del s. XVI.

Otro aspecto que pudimos observar, este menos documentado, es que los fondos de los casetones están formados por dos piezas de piedra de tamaño semejante a un sillarete, pieza prismáticas habitualmente usadas durante el siglo XV e inicios del XVI. Estas piezas quedan labradas sólo por su cara vista, mostrando una gran irregularidad por su trasdós. Esta solución es habitual en las bóvedas tardogóticas donde la plementería o superficie de sillares entre nervios está formada igual que los casetones que aquí encontramos. De este modo la escasez de labra del trasdós responde a un ahorro de tiempo de ejecución, pero también es un modo de facilitar la homogeneidad en el funcionamiento de piezas tan dispares, pues al verter un derretido de mortero de cal que penetraba entre los huecos, logramos formar un cuerpo bastante homogéneo con los sillares usando un conjunto de materiales semejantes. La calidad de la cal disponible en Morón es excepcional, lo que garantizaba su presencia en la obra desde el inicio de las reformas emprendidas a inicios del s. XVI. Sólo las fuertes tracciones producidas a consecuencia del desplome del muro exterior de la capilla alteró esta unidad constructiva en sentido transversal al movimiento. Además de documentar gráfica y fotográficamente este desgarro, encontramos de nuevo otro indicio del uso de sistemas tradicionales en formalizaciones nuevas que indican la forma conservadora de proceder de estos primeros maestros del renacimiento, como ya nos anunciara Palacios (21).

Al ser retirada la edificación posterior en los años setenta del pasado siglo, se alteró definitivamente el estado de equilibrio que la había mantenido en pie. El terreno excavado en exceso debilitó la respuesta del estribo a las cargas que procedían de las bóvedas del tramo de la nave principal, alterando la configuración del arbotante hasta producir una descargar del arco que lo estructura, sobre el que también tuvimos que intervenir. El exceso de solicitaciones afectó a la composición interna del estribo en las zonas donde había sido horadado para recibir al muro de la casa y los maderos de las vigas de sus forjados. Esta deformación produjo el desplazamiento de los aplacados laterales de la capilla, que unido a los movimientos del propio estribo, modificaron las condiciones de estabilidad de la bóveda, cuyos nervios y cruceros se separaron hasta llegar al estado anterior al apuntalamiento realizado al final del pasado siglo.

\section{CONCLUSIONES}

Patrocinada por un miembro de la nobleza local, imbuido por la nueva corriente artística y de pensamiento humanista, localizamos este pequeño edificio en una encrucijada de caminos donde se encuentran las tradiciones adquiridas en el ámbito de lo constructivo y tecnológico, con las nuevas aspiraciones formales y simbólicas de un colectivo que ve en estas empresas una manera de reivindicar su rol social. Para responder constructivamente a estos nuevos retos se usó tanto de la tecnología heredada de los antiguos maestros medievales, como de los nuevos avances que la ciencia y la técnica aportaban. Así, tanto la configuración de la portada con las dos pilastras jónicas, entablamento y frontón, como la bóveda en forma de baída artesonada sobre paramentos lisos, recurrirán a sistemas constructivos tradicionales, a pesar de configurarse como elementos formales novedosos.

La bóveda que hemos analizado, quizás el elemento más complejo en su ejecución y más simple en su apariencia visual, no puede entenderse al margen de esa necesidad de experimentar nuevas formas, ni de la tradición que la arropa. Más aún, es el dominio de unos sistemas de trazado y construcción fuertemente arraigada en la tradición lo que permite la libertad en el uso de los recursos necesarios para la experimentación. Esta situación fue muy frecuente a lo largo del territorio peninsular durante los años centrales del siglo XVI, dando origen en el tiempo a lo que conocemos como la moderna estereotomía de la piedra, considerada una de las peculiaridades más destacables de nuestra arquitectura en el tránsito a la edad moderna.

El aumento de la complejidad en la ejecución del trazado de las bóvedas renacentistas en piedra irá requiriendo sistemáti- 
camente novedades geométricas para su control formal, que acabarán por afectar tanto a su diseño como a su construcción, dando entrada a las bóvedas baídas de superficies uniformes poco tiempo después. En este contexto, la utilización del tipo de bóveda analizado tiene una vida muy corta, pues a mediados del siglo XVI prácticamente desaparecen, quedando como testigos de un intenso momento de cambios, como un lugar límite entre el gótico y el renacimiento. Las respuestas gráficas y constructivas a las nuevas exigencias formales que se inician con este tipo de arquitectura y sus formas de construir, serán el eslabón entre la tradición medieval y el germen de la estereotomía moderna, que desembocarán en la posterior disciplina que conocemos como geometría descriptiva, y a través de ella, de los sistemas básicos de representación en el plano. Sistema que permitió el avance de la arquitectura y la ingeniería hasta la aparición de los soportes digitales.

Algunas conclusiones particulares:

1. En cuanto a la metodología de trabajo, entendemos que el proceso de intervención es un momento irrepetible de aprendizaje donde entramos en contacto con la parte más íntima de los edificios históricos, donde se expresan en todo su pragmatismo los procedimientos constructivos de cada época, lo que viene a denominarse "el saber constructivo". La propia intervención nos sitúa en un lugar privilegiado desde el que observar ese difícil equilibrio entre los requerimientos del arte y la técnica, de la forma y la función, o del sentimiento y la razón (22). Pero por desgracia, debido a la cada vez más acuciante carencia de medios, este momento de aprendizaje suele estar atropellado por los tiempos que impone la ajustada planificación de la obra o por las cada vez mayores exigencias normativas que condicionan la redacción de los estudios previos y los propios proyectos de intervención. Por ello entendemos muy importante el desarrollo de un trabajo sistemático de registro, toma de datos y análisis previos y paralelos a la intervención como instrumentos para su correcta valoración.

2. En cuanto a las soluciones adoptadas en la reforma, queda patente el descuido en la solución de los aplacados, más pendientes de lo ornamental que de lo constructivo, seguramente por la novedad de lo primero y lo convencional de lo segundo. En otras obras más maduras ambas cuestiones volverán a integrarse, adquiriendo un sentido de unidad forma-construcción que será un aspecto destacable de nuestra arquitectura renacentista de mediados de siglo XVI. Así se mostrará en la obra de Hernán Ruiz el joven, maestro que relevará a Gainza en la maestría del obispado y en obras como la que estamos analizando. La composición del muro en tres hojas o capas (dos de sillares y una interior de calicostrado), permite una manipulación de las exteriores para introducir elementos decorativos como portadas, tabernáculos, etc, confiando en exceso la unidad estructural al relleno interior.

3. Por último, la trabazón de las fábricas queda confiada prácticamente a las capas externas, provocan un simple adosamiento de los rellenos interiores. Cualquier movimiento de la estructura afecta directamente a estas capas externas que han quedado muy reducidas de potencia. Si estas capas son débiles, como es el caso de esta capilla, provocan desgarros importantes, pues la capa interior no contribuye a la unidad estructural. Las modificaciones exteriores del subsuelo, la inestabilidad de las rocas de yeso presentes en el mismo y las filtraciones superiores a través de la cubierta, agravan los efectos de las deformaciones estructurales. En elementos tan exteriores y límites como esta capilla, es difícil que los elementos estructurales se reorganicen buscando nuevos estados de equilibrio, Ilegando a la situación que nos encontramos. Los procesos de sventramento eliminan estructuras que históricamente han ido creando estos reajustes, pudiendo provocar el colapso en caso de no consideramos sus efectos estructurales.

\section{AGRADECIMIENTOS}

A José María Guerrero Vega, compañero en este trabajo de restauración, a Juan Carlos Gómez de Cózar por su colaboración en el análisis estructural realizado como parte de los trabajos previos a la intervención, al Grupo de Investigación HUM 799 (Estrategias de Conocimiento Patrimonial), cuyo método de investigación está presente en las aportaciones realizadas. A la empresa constructora Sanor S. L. y a su buen hacer $y$ al de sus trabajadores en este proyecto. Y por supuesto, al comité de redacción de esta revista y los acertados comentarios de sus evaluadores. El contenido de esta aportación forma parte de la participación en el proyecto I+D (BIA2009-14350) "Construcción en piedra de cantería en los ámbitos mediterráneo y atlántico. Análisis de ejemplos construidos", dirigido por Enrique Rabasa. 


\section{REFERENCIAS}

(1) Choisy, A. (1899). Histoire de I'architecture, Paris. Gauthier-Villars. Versión en casteIlano consultada, Historia de la Arquitectura. Victor-Lerú. (1963) Buenos Aires.

(2) Fernández Muñoz, Á.L. (1992). El proyecto como condición de la restauración arquitectónica. Restauración Arquitectónica. 1: 137-148. Univ. Valladolid. Valladolid.

(3) Palacios Gonzalo, J.C. (1990). Trazas y cortes de cantería en el Renacimiento español. Ministerio de Cultura. ICRBC. Madrid.

(4) Gómez Martínez, J. (1998). El gótico español de la edad moderna. Las bóvedas de crucería. Univ. de Valladolid. Valladolid.

(5) Rabasa Díez, E. (1996). Técnicas góticas y renacentistas en el trazado y talla de las bóvedas de crucería españolas del s. XVI. Historia de la Construcción. Actas del primer congreso Nacional. Instituto Juan de Herrera/CEHOPU, pp. 423-433. Madrid. Forma y construcción en piedra. De la cantería medieval a la estereotomía del siglo XIX. Akal. Madrid 2000.

(6) Proyecto de Investigación del Ministerio de Ciencia e Innovación del Gobierno de España (BIA2009-14350) Construcción en piedra de cantería en los ámbitos mediterráneo y atlántico. Análisis de ejemplos construidos, dirigido por Enrique Rabasa. En este proyecto participo como miembro del equipo de investigadores.

(7) Palacios González, J.C y Bravo Guerrero, C. (2012). Construction of a Pendentive Grid Crossing Vault. Nuts and Bolts of Construction History. 1: 81-88. Edit A. et J. Picard. París.

(8) Pinto Puerto, F. (2001). Las esferas de piedra. Sevilla como lugar de encuentro entre arte y ciencia del renacimiento. Diputación de Sevilla. Sevilla.

(9) Rodríguez Estevez, J.C. (2011). Martín de Gainza (ca.1505-1556). Artistas Andaluces y artífices del arte andaluz. pp. 255-288. Publicaciones Comunitarias. Grupo Hércules. Sevilla.

(10) Proyecto de Investigación del Ministerio de Ciencia e Innovación, Gobierno de España. (HAR2011-25138): Arquitectura Tardogótica en la Corona de Castilla: Trayectorias e Intercambios. Dirigido por Begoña Alonso. www.tardogotico.es.

(11) Morón de Castro, M.F. (1995). La iglesia de San Miguel. Cinco siglos en la historia de Morón de la Frontera. XIV-XVII. Univ. Sevilla, Sevilla.

(12) Fernández Naranjo, J.A. (2007). Fascículus. La Piedra Postrera. pp. 307-325. Cabildo Metropolitano. Sevilla.

(13) Rodríguez Estévez, J.C. (2007). El gótico catedralicio. La influencia de la Catedral en el Arzobispado de Sevilla. La Piedra Postrera. pp. 175-257. Cabildo Metropolitano. Sevilla.

(14) Gómez de Cózar, J.C. (2009). Cul de Lampe: Adaptación y disolución del gótico en el Reino de Sevilla. pp. 73-83. Secretariado de Publicaciones de la Universidad de Sevilla. IUACC. Sevilla.

(15) Pinto Puerto, F., y Guerrero Vega, J.M. (2009). Estudios previos a la intervención en la capilla de la Antigua. Iglesia de San Miguel, en Morón de la Frontera (Sevilla). Arqueología de la Arquitectura, 6: 267-287.

(16) Informe geotécnico realizado por la empresa Vorsevi con expediente 40040561. (2010).

(17) Amores Carredano, F. y Chisvert Jiménez, N. (1993). Tipología de la cerámica común bajo medieval y moderna sevillana (SS.XV-XVIII) I, La loza quebrada de relleno de bóvedas. Revista de prehistoria y arqueología Spal, 2: 269-325.

(18) Gómez de Cózar, J.C y Benítez Bodes, R. (2008). Las soluciones inconclusas de las cubiertas de los templos medievales del Reino de Sevilla, España. Informes de la Construcción, 60(509): 57- 67. doi:10.3989/ic.2008.v60.i509

(19) Jiménez Sancho, A. (2000). Rellenos cerámicos en las bóvedas de la Catedral de SeviIla. Actas del III Congreso Nacional de Historia de la Construcción. 2: 561-568. Instituto Juan de Herrera. Madrid.

(20) Vandelvira, Alonso. Libro de trazas de cortes de piedra. 1575 y 1591 . Hemos usado la edición facsímil de Barbé-Coquelin de Lisle, G.: El tratado de Arquitectura de Alonso de Vandelvira. 2 vol. Caja de Ahorros Provincial de Albacete. Albacete, 1977.

(21) Palacios Gonzalo, J.C. (1990). Trazas y cortes de cantería en el Renacimiento español. Ministerio de Cultura, p. 226-233. ICRBC. Madrid.

(22) González Moreno-Navarro, J.L. (1993). El legado oculto de Vitruvio. p. 18, Alianza Forma. Madrid. 